Check for updates

Cite this: RSC Adv., 2018, 8, 32621

Received 28th July 2018

Accepted 12th September 2018

DOI: $10.1039 / c 8 r a 06362 a$

rsc.li/rsc-advances

\section{Formulation, characterization and evaluation of morusin loaded niosomes for potentiation of anticancer therapy}

\author{
Srishti Agarwal, ${ }^{a}$ M. Sheikh Mohamed, ${ }^{a}$ Sreejith Raveendran, ${ }^{b}$ Ankit K. Rochani, ${ }^{c}$ \\ Toru Maekawa ${ }^{a}$ and D. Sakthi Kumar (iD *a
}

\begin{abstract}
Morusin, a water-insoluble prenylated flavonoid is known for its numerous medicinal properties. It manifests its anticancer potential by suppression of genes involved in tumor progression. However, poor solubility of the drug results in low bioavailability and rapid degradation thus hindering its clinical utilization. In order to overcome this, we have synthesized a niosome system composed of non-ionic surfactant span 60 and cholesterol using a thin-layer evaporation technique to improve the aqueousphase solubility of the drug. Highly cytocompatible niosomes of $479 \mathrm{~nm}$ average size with smooth and uniform spherical morphology were synthesized in a facile manner. Unlike free morusin, nanomorusin was found to be freely dispersible in aqueous media. Having an extremely high drug entrapment efficiency $(97 \%)$, controlled and sustained release of morusin resulting in enhanced therapeutic efficacy was observed in cancer cell lines of 4 different lineages. The results demonstrate that the morusinniosome system is a promising strategy for enhanced anti-cancer activity against multiple cancer types and could be an indispensable tool for future targeted chemotherapeutic strategies.
\end{abstract}

\section{Introduction}

Cancer remains the principal cause of death in the world, regardless of economic or financial status of the respective nations. ${ }^{1}$ Clinical cancer treatment is heavily dependent upon chemotherapy in conjunction with radiation therapy and surgery, with immunotherapy playing a supportive role. Despite major advances in these treatment modules, high instances of tumor relapse and long-term permanent side effects remain unaddressed. Generally, in order to impede the growth of proliferating tumor cells, cytotoxic drugs are given which deter the proliferation of cancer cells, but play a significant role in non-target toxicity resulting in unwanted and severe side effects.

Therefore, extensive research is being carried out on using substances derived from natural origins as potent inhibitors of cancer, which have roots in numerous traditional medicinal regimes. Natural substances like curcumin, ${ }^{2,3}$ resveratrol, ${ }^{4,5}$ quercetin, ${ }^{6,7}$ and green tea catechins ${ }^{8,9}$ have displayed considerable toxicity against various cancer types with minimal non-

\footnotetext{
${ }^{a}$ Bio Nano Electronics Research Center, Graduate School of Interdisciplinary New Science, Toyo University, Kawagoe, Saitama, 350-8585, Japan. E-mail: sakthi@toyo. jp; Fax: +81-366-77-1140; Tel: +81-492-39-1636/1375/1640

${ }^{b}$ School of Pharmacy and Biomolecular Sciences, University of Brighton, Moulsecoomb, Brighton, BN2 4GJ, UK

'Jefferson College of Pharmacy, Department of Pharmaceutical Science, Thomas Jefferson University, 1020 Locust Street, Philadelphia, PA-19107, USA
}

target toxicity. However, with the rise of therapies that utilize high throughput screening methods for production of pharmaceutical molecules and drugs, the interest in natural products has declined rapidly. ${ }^{10}$

Despite showing tremendous results against few cancer types, these therapies remained unsatisfactory for many solid cancers due to undesired side-effects, which redirected the attention on natural products. Rapamycin, produced by the soil bacterium Streptomyces hygroscopicus was the first anticancer natural product to be approved in 2007 followed by other natural product derivatives. ${ }^{10,11}$ Being chemoprotective in nature, these products are classified either as potent antioxidants, phenolic or having reactive groups, which impart protective properties. ${ }^{12}$

One such natural product is morusin, a prenylated flavonoid derived from the root bark of Morus alba which has been stated to possess anti-inflammatory, anti-oxidant and anti-bacterial activities. ${ }^{13}$ Effectiveness of morusin as a potent anti-tumor agent has been elucidated by a number of studies. Various biological activities displayed by morusin include protection against nitric oxide (NO)-induced neuroblastoma cell death, ${ }^{14}$ scavenging activity against superoxide anion radicals, ${ }^{15}$ antidiabetic activity, ${ }^{16}$ adipocyte differentiation, ${ }^{17}$ etc. Predominantly, morusin has shown cytotoxic effects against cancer cell lines including breast adenocarcinoma, ${ }^{18,19}$ colorectal adenocarcinoma, ${ }^{20}$ gastric cancer, ${ }^{21}$ etc. Recent studies have reported inhibition of NF-KB and STAT-3 signaling by morusin in prostrate, pancreatic and liver cancer cells. ${ }^{22-24}$ In addition, morusin 
is also known to inhibit the expression of metalloproteinases (MMP-2 and MMP-9), which are elevated in the tumor microenvironment. ${ }^{\mathbf{2 4 , 2 5}}$ These evidences though support morusin as an effective natural anticancer drug; its full potential has not been explored mainly due to its poor aqueous solubility and bioavailability. Hydrophobicity of therapeutic moieties is a major issue in drug formulations, which results in rapid clearance by the reticuloendothelial system (RES) and poor bioavailability, restricting their full clinical potential. Additionally, non-specific accumulation of the toxic compounds cannot be monitored or controlled, leading to side effects.

Nanotechnology offers solutions for such limitations by providing a stable reservoir for hydrophobic compounds, and also minimizing off-site interactions, apart from assisting in escaping the RES and reaching the target cells with high precision. $^{26}$ Numerous examples of nano-medicine formulations exist in literature $\mathrm{e}^{27-30}$ with continuous updates on a near to daily basis. Of these, micellar systems have attracted much attention due to their unique properties and enhanced biocompatibility. These are a class of colloidal nanocarriers comprising liposomes or niosomes, which are known to possess significant advantages over conventional dosage forms. ${ }^{31}$ Niosomes are novel drug delivery carriers having a bilayer that can either be unilamellar, or bi-lamellar composed of various non-ionic surfactants and cholesterol. Due to the availability of a variety of non-ionic surfactants, the vesicles can be designed accordingly. ${ }^{32}$ Compared to other surfactants, spans and tweens are known to have higher entrapment efficiency and better release characteristics. ${ }^{33,34}$ Niosomes offer prime advantages like ease of preparation, reduced toxicity, increased stability, protection against degradation of active drug molecules, and enhanced bioavailability compared to liposomes, which have issues with physical and chemical stabilities. ${ }^{32,35,36}$ Due to their unique and desirable properties, niosomes have been in use as a part of the cosmetic industry since $1970 .{ }^{37}$ The drugs can either be entrapped in aqueous core (hydrophilic) or bilayer (hydrophobic) due to presence of two distinct types of microenvironments. ${ }^{36}$ Drug delivery using niosomes has been achieved using different modes of administration like intramuscular, ${ }^{38}$ intravenous, ${ }^{39}$ transdermal, ${ }^{40}$ etc. and niosomes have been employed for delivery of various chemotherapeutic drugs like curcumin, ${ }^{36}$ cisplatin, ${ }^{41}$ doxorubicin, ${ }^{42}$ paclitaxel,,${ }^{43}$ etc. It has been specified that entrapment of drugs inside a niosomal nano carrier can prevent the fluctuations in drug plasma concentration thus achieving controlled drug delivery and reduced systemic toxicity of anticancer drugs. ${ }^{31}$

As far to our knowledge, there is no study reporting morusin as a nano formulation for curbing cancer growth. The present study was formulated to obtain a robust drug delivery system for encapsulating morusin in nanoparticles and accordingly, a facile synthesis route was employed for producing morusin niosomes and their cytotoxic and cellular localization studies performed to assess the feasibility of this nanoformulation as a potential anti-cancer candidate. We also performed docking studies to observe binding of morusin to matrix metalloproteinases (MMP-2) and (MMP-9) which are up regulated in the tumor milieu in pancreatic cancer, ${ }^{\mathbf{4 4}}$ colorectal cancer, ${ }^{\mathbf{4 5}}$ ovarian cancer ${ }^{\mathbf{4 6 , 4 7}}$ and breast cancer, ${ }^{\mathbf{4 8 , 4 9}}$ etc. thus contributing to tumor progression and metastasis.

\section{Experimental section}

\section{Materials}

Span 60 (sorbitan monostearate) and cholesterol were procured from Tokyo chemical industry Co. Ltd. Morusin was purchased from Chemfaces. Chloroform was purchased from Kanto chemicals, Japan. Human triple negative breast cancer (MDAMB-453), colorectal adenocarcinoma (HT-29), ovarian cancer (SKOV-3), pancreatic cancer (PANC-1) cell lines and Mc-coy medium were purchased from American Type Culture Collection (ATCC), USA. Mouse fibroblast cell lines (L929) was purchased from Riken BioResource Center, Japan. Dulbecco's modified Eagle's medium (DMEM), trypsin (0.25\%), fetal bovine serum (FBS), penicillin $\left(5000 \mu \mathrm{g} \mathrm{m} \mathrm{m}^{-1}\right) /$ streptomycin $(5000 \mu \mathrm{g}$ $\mathrm{ml}^{-1}$ ) and phosphate buffer saline (PBS) were procured from Gibco (Life Technologies). Alamar blue cell viability kit reagent was purchased from Invitrogen, USA. Live/dead viability/ cytotoxicity kit, Leibovitz media, Lysotracker deep red and Nuc blue live ready probes reagent were acquired form Thermo Fisher Scientific. All other chemicals and solvents were of analytical grade.

\section{Methods}

Preparation of niosomes. Drug loaded niosomes were synthesized using thin layer evaporation method with slight modifications. ${ }^{32}$ Briefly, $100 \mathrm{mg}$ of span $60,20 \mathrm{mg}$ of cholesterol and $10 \mathrm{mg}$ of drug (morusin) were dissolved in $20 \mathrm{ml}$ of chloroform. The solution was kept for intermittent stirring with a magnetic stirrer for $1 \mathrm{~h}$. After $1 \mathrm{~h}$, the resultant solution was kept for evaporation in a rotary evaporator for $1 \mathrm{~h}$, which results in formation of a thin film. In order to get a completely dried film the flask was kept in a vacuum desiccator for $1 \mathrm{~h}$. The obtained thin film was then hydrated using $20 \mathrm{ml}$ of ultrapure distilled water and 4 cycles of heating and vortexing $(3 \mathrm{~min}$ each) were performed in order to remove any residual chloroform from the solution. The resulting solution was then centrifuged at $8000 \mathrm{rpm}$ for 30 minutes for atleast 3 cycles in order to concentrate down the particles. After discarding the supernatant, the pellet was washed with ultra pure water thrice. The product mor-nio (morusin-niosomes) was freeze dried and stored at $-20{ }^{\circ} \mathrm{C}$ till further use. Void nio (devoid of morusin) and C- 6 nio (coumarin-6 loaded niosomes for cellular uptake studies) were also synthesized similarly.

Determination of yield, entrapment and loading efficiencies. The yield of niosomes was calculated by using the following eqn $(1)^{50}$

$$
\% \text { Yield }=\frac{\text { dry weight of the nanoparticles obtained }}{\left(W_{\mathrm{m}}+W_{\text {span } 60}+W_{\text {cholesterol }}\right)} \times 100
$$

where, $W_{\mathrm{m}}$ - weight of morusin, $W_{\text {span } 60}$ - weight of span 60, $W_{\text {cholestrol }}$ - weight of cholesterol, used for niosome synthesis. 
The UV-Vis absorption spectra of free morusin, void nio and mor-nio was recorded by UV-Vis spectrophotometer (DU730, Beckman Coulter) for confirming entrapment of morusin. The amount of free drug released in the supernatant was then measured quantitatively against the calibration curve of the drug and the unknown quantity was determined. Percentage of entrapment efficiency was determined with a spectrophotometer using the eqn $(2)^{50}$

$$
\% \text { Entrapment }=\frac{C_{\mathrm{i}}-C_{\mathrm{f}}}{C_{\mathrm{i}}} \times 100
$$

where, $C_{\mathrm{i}}$ is the initial drug concentration used for preparation of niosomes and $C_{\mathrm{f}}$ is the concentration of free drug in the supernatant obtained upon washing.

Loading efficiency was determined using the formula (3) ${ }^{51}$

$$
\% \text { Loading }=\frac{\text { amount of drug in nanoparticles }}{\text { gross weight of nanoparticles }} \times 100
$$

Niosome characterization. The size and morphological analysis of the synthesized nanoparticles was done using Hitachi SU6600 scanning electron microscope (SEM) and JEOL JEM-2100 transmission electron microscope (TEM). Mor-nio and void nio were dispersed in milliQ water for executing all characterization experiments. For doing SEM analysis, $50 \mu \mathrm{l}$ of water-dispersed niosomes was dropped on a clean glass substrate and vacuum dried. The dried sample was sputter coated with platinum for 35 seconds (Hitachi E-1030, ion sputter) and observed under SEM functioning at an accelerating voltage of $10 \mathrm{kV}$. 100-125 individual particle diameters were measured and the values were averaged to find the mean diameter value of the resulting particles. For TEM, $10 \mu \mathrm{l}$ of sample was placed on a hydrophilized $\mathrm{Cu}$ microgrid and airdried with observations performed at an accelerating voltage of $100 \mathrm{kV}$. Particle size distribution and zeta-potential were measured using zetasizer (Malvern, Nano-ZS). For particle size distribution analysis, disposable polystyrene cuvettes were used and the average diameter and polydispersity index (PDI) values were acquired at $25{ }^{\circ} \mathrm{C}$. Zeta potential measurements were carried out at $25{ }^{\circ} \mathrm{C}$ using a dip cell. All size and zeta potential measurements were carried out in triplicates.

In order to characterize the structure and explore the chemical bonding patterns between morusin and the niosome components (span 60 and cholesterol) Fourier Transform InfraRed Spectroscopy (FTIR) (Thermo Scientific, Nicolet iS50 FT-IR) was performed. Samples were made into pellets using potassium bromide $(\mathrm{KBr})$ and subjected for analysis under transmittance mode $\left(4000-500^{-1} \mathrm{~cm}\right)$.

In vitro drug release. In order to study the diffusion of morusin from niosomes, drug release studies were carried out. The nanoparticles were dispersed in phosphate saline buffer (PBS) at two different $\mathrm{pH}$ conditions of $\mathrm{pH} 4.5$ and $\mathrm{pH}$ 7.4. All experiments were carried out in triplicates. $15 \mathrm{mg}$ of sample was dispersed in $15 \mathrm{ml}$ of PBS buffer and distributed in 15 Eppendorf tubes such that concentration per tube was $1 \mathrm{mg} \mathrm{ml}^{-1}$. All tubes were kept at $37^{\circ} \mathrm{C}$ under a continuous shaking of $130 \mathrm{rpm}$ for 9 days. At predetermined time intervals, one tube was taken out and centrifuged at $15000 \mathrm{rpm} / 30 \mathrm{~min}$. Absorbance of the resulting supernatant was read by a UV-Vis spectrophotometer at fixed wavelength of the drug i.e. $269 \mathrm{~nm}$ and the amount of morusin released was determined. The release kinetics was calculated according to the following eqn $(4)^{52}$

$$
\% \text { Release }=\frac{\text { released drug }}{\text { total drug }} \times 100
$$

where 'released drug' is the concentration of the drug released and collected at pre-defined time intervals and 'total drug' is the amount of drug entrapped in the nanoparticles.

Cell culture. Human triple negative breast cancer (MDA-MB453), Human colorectal adenocarcinoma (HT-29), Human pancreas ductal adenocarcinoma (PANC-1), Human ovarian cancer (SKOV-3) and mouse fibroblast cell lines (L929) were chosen to conduct in vitro cell studies and analyze the therapeutic efficacy of mor-nio. PANC-1 cells along with SKOV-3 and L929 were cultured in T-25 tissue culture flasks in DMEM, HT29 in Mc-coy and MDA-MB-453 cells in Leibovitz media, supplemented with $10 \% \mathrm{FBS}, 1 \%$ penicillin/streptomycin at $37{ }^{\circ} \mathrm{C}$ in a humidified atmosphere of $5 \% \mathrm{CO}_{2}$ and for MDA-MB-453 without $\mathrm{CO}_{2}$ till cells were $80 \%$ confluent. The cells were sub cultured every 3-4 days or once the confluency was attained.

Cytocompatibility analysis. To assess whether the bare niosomes (void nio) exert any toxicity, cytocompatibility analysis was performed. For the assay, confluent cells were trypsinized, plated in a microtiter 96-well plate at a density of $8 \times 10^{3}$ cells per $\mathrm{ml}$ and maintained at $37{ }^{\circ} \mathrm{C}, 5 \% \mathrm{CO}_{2}$ and for MDA-MB-453 without $\mathrm{CO}_{2}$. After incubation of $24 \mathrm{~h}$, media was replenished and void niosomes were added in the concentration range of 50 $\mu \mathrm{g} \mathrm{ml}^{-1}, 100 \mu \mathrm{g} \mathrm{ml}^{-1}, 250 \mu \mathrm{g} \mathrm{ml}^{-1}, 500 \mu \mathrm{g} \mathrm{ml}^{-1}$, and $1000 \mu \mathrm{g}$ $\mathrm{ml}^{-1}$. Cells without nanoparticles were considered as controls. After treatment with the desired concentration of void nio, the cells were incubated for 6,24 and 48 hours under respective culture conditions. After defined incubation period, $10 \%$ alamar blue dye (which monitors the reducing environment of healthy cells and converts the non-fluorescent resazurin (blue color) to a highly fluorescent reduced form resorufin (red color) indicative of metabolic activity of cells) was added to the cells and plates were incubated for 4 hours following which fluorescence was recorded at $580-610 \mathrm{~nm}$ by a microplate reader (Power scan HT Microplate reader, Dainippon Sumitomo Pharma, Japan).

In vitro cytotoxicity studies. The therapeutic efficacy of mornio on different cancer cell lineages and normal cells was assessed using alamar blue. The assay procedure was similar to previous section. After incubation of cells for $24 \mathrm{~h}$, media was replenished and free drug and mor-nio were added at varying concentration $\left(50 \mu \mathrm{g} \mathrm{ml}^{-1}, 100 \mu \mathrm{g} \mathrm{ml}^{-1}, 250 \mu \mathrm{g} \mathrm{ml}^{-1}, 500 \mu \mathrm{g}\right.$ $\mathrm{ml}^{-1}$, and $1000 \mu \mathrm{g} \mathrm{ml} \mathrm{m}^{-1}$ ). The culture/data acquisition conditions and parameters were similar as described in previous section. Here we used free morusin in 0.5\% DMSO, mor-nio and untreated cells as control group for the experiment. The assay was performed in three autonomous experiments all in triplicates and cell viability was computed using the formula $(5)^{51}$ 


$$
\% \text { Cell viability }=\frac{A_{\text {sample }}}{A_{\text {control }}} \times 100
$$

where $A_{\text {sample }}$ is the absorbance of the sample and $A_{\text {control }}$ is the absorbance of the control.

Internalization and co-localization of niosomes. The internalization of niosomes in L929, HT-29, PANC-1, SKOV-3 and MDA-MB-453 was studied by confocal microscopy. As morusin is non-fluorescent, C- 6 niosomes were utilized for the assay. The cells were grown in respective growth media/culture condition on $35 \mathrm{~mm}$ glass base culture dishes at a concentration of approximately $5 \times 10^{4}$ cells for $24 \mathrm{~h}$ following which $100 \mu \mathrm{g}$ $\mathrm{ml}^{-1}$ of C- 6 nio was subsequently added to the cultures and incubated for 2 hours. After incubation, the cells were washed with $1 \times, \mathrm{pH} 7.4$ PBS buffer to remove any excess unbound particles. Lysotracker deep red and Nuc blue were then added to the plates in order to stain the acidic compartments and nuclear region, respectively according to product instructions. The cells were examined using a high-speed confocal laser-scanning microscope (CLSM, Nikon A1 plus Tokyo, Japan) at excitation wavelengths of $402 \mathrm{~nm}$ (NucBlue), $488 \mathrm{~nm}$ (C6) and $630 \mathrm{~nm}$ (Lysotracker).

Live/dead assay. The qualitative determination of live and dead cells after nanoparticle treatment was carried out using CLSM. Cells at a density of $5 \times 10^{4}$ cells were plated in a $35 \mathrm{~mm}$ glass base dishes for $24 \mathrm{~h}$ following which $500 \mu \mathrm{g} \mathrm{ml}^{-1}$ of free morusin, void nio, and mor-nio were added and incubated for a further $24 \mathrm{~h}$ period. Post-incubation, cells were washed twice with PBS and stained with live/dead viability/cytotoxicity reagent as per product instructions. The plates were then incubated at RT for $1 \mathrm{~h}$ following which the cells were observed in CLSM at excitation and emission wavelengths of 495/515 nm for calcein and 528/617 for EthD-1. The number of dead and viable cells in the test was compared to the total number of viable cells in the control.

Docking studies. In order to check the probable binding of morusin to MMP-2 and MMP-9, molecular docking was executed using Autodock 4.0 software (USA). For performing Docking simulations, we downloaded crystal structures of proteins MMP9 (PDB id: 1GKC) and MMP2 (PDB id: 1CK 7) from protein databank (pdb). Structure of ligand (Morusin) was downloaded from PubChem (PubChem id: 5281671). Both ligand and protein structures were subjected to geometry and charge optimization using MMFF94 and Gasteiger charge calculations. Essential hydrogen atoms, Kollman united atom type charges, and solvation parameters were added with the aid of AutoDock tools. Affinity (grid) maps of $100 \times 100 \times 100 \AA$ grid points and $0.375 \AA$ spacing were produced using the Autogrid program. AutoDock parameter set-and distancedependent dielectric functions were used in the computation of the van der Waals and the electrostatic terms, respectively. Docking simulations were accomplished using the Lamarckian genetic algorithm (LGA) and the Solis \& Wets local search method. Initial position, orientation, and torsions of the ligand molecules were set randomly. All rotatable torsions were released during docking. Each docking experiment was derived from 100 different runs that were set to terminate after a maximum of 2500000 energy evaluations. The population size was set to 150 . During the search, a translational step of 0.2 $\AA$, and quaternion and torsion steps of 5 were applied. We selected ligand-protein complexes that offered most stable conformations based on lowest Gibbs binding energy. Short range van der Waal and electrostatic interactions, hydrogen bonding, entropy losses were included for energy-based autodock scoring function (Morris et al., 1998). The results were downloaded in pdb files and subjected to analysis using Drug Discovery Studios software.

\section{Results and discussion}

\section{Morphological characterization of morusin loaded niosomes}

Niosomes (void nio/mor-nio) were synthesized using thin-layer evaporation method in order to enhance the bioavailability, efficacy and aqueous solubility of the drug. Span 60 was used as an ionic surfactant and cholesterol as an additive for preparation of niosomes. Cholesterol imparts rigidity ${ }^{53}$ while span 60 protects the drug from proteolytic enzymes leading to higher physical stability. ${ }^{\mathbf{5 4 5 5}}$ The synthesized niosomes were then characterized for their morphology, size distribution and surface characteristics.

As shown in SEM micrograph (Fig. 1a and d), the void nio (445 $\mathrm{nm}$ ) and mor-nio (479 $\mathrm{nm}$ ) have a uniform spherical morphology with smooth surface characteristics. The vesicles are formed due to self-assembly of the non-ionic surfactants upon their interaction with the aqueous medium leading to creation of a concentric bilayer structure having morphology comparable to liposomes but without phospholipids which imparts better stability. ${ }^{35,56}$ Similar observations were made in TEM as well where smooth and spherical niosomes could be visualized (Fig. 1b and e). Fig. 1c and f show Gaussian size distribution of the void and mor-loaded nanoparticles wherein majority particles in both cases lie in the size range of 400$500 \mathrm{~nm}$. Detailed characterization of the particles is provided in Table 1. Further, dynamic light scattering (DLS) measurement done for void and mor-nio confirmed nanoparticles with size distribution in the range of $400-500 \mathrm{~nm}$ and z-average size of $445 \mathrm{~nm}$ and $479 \mathrm{~nm}$ which correlated with SEM and TEM observations. Since, the approximate size of vesicles/micelles for cellular uptake via the endocytic pathway is $100-500 \mathrm{~nm}$, our synthesized mor-nio fall in the desired range for successful internalization by cancer cells. ${ }^{57,58}$

The cellular interaction and uptake of nanoparticles is highly influenced by the surface charge of nanoparticles. ${ }^{59}$ Studies have reported that negatively or positively charged nanoparticles are more readily internalized as compared to their uncharged counterparts. ${ }^{59}$ Although positively charged nanoparticles have a rapid cellular uptake in comparison to negatively charged nanoparticles, they are known to cause hemolytic size-effects and cytotoxicity to normal tissues. ${ }^{\mathbf{5 9 , 6 0}}$ The synthesized void and mor-nio had a zeta potential of -14.2 and -19.8 , respectively, denoting the stability of the colloidal suspension of niosomes. This could be accredited to the coulombic repulsion forces occurring from their surface charge, which overcomes the van der Waals attractive forces 

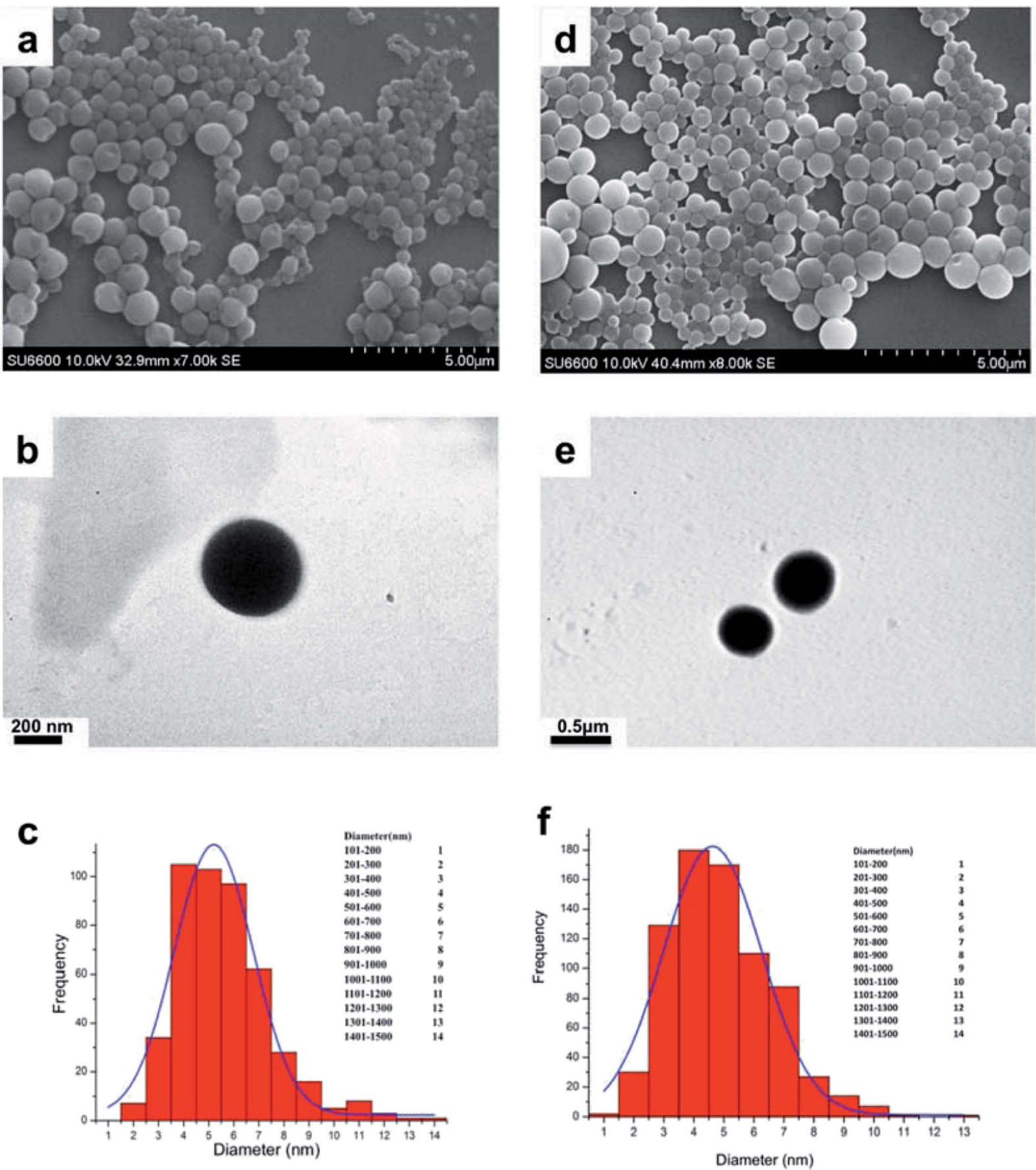

Fig. 1 (a) SEM micrograph of void nio (d) SEM micrograph of mor-nio. (b) TEM image of void nio (e) TEM image of mor-nio (c and f) Gaussian size distribution for void and mor-nio.

Table 1 Physico-chemical characterization of void and mor-nio

\begin{tabular}{lll}
\hline Characterization parameter & Void nio & Mor-nio \\
\hline$Z$ average diameter (nm) & 445 & 479 \\
Poly dispersity index (PDI) & 0.3 & 0.29 \\
Zeta potential (mV) & -14.2 & -19.8 \\
Drug loading & - & $7.69 \pm 0.14 \%$ \\
Entrapment efficiency & - & $97 \pm 1.25 \%$ \\
\% Yield & $38.46 \pm 1.31 \%$ & $31.07 \pm 2.43 \%$
\end{tabular}

between them. ${ }^{50}$ Table 1 depicts the physico-chemical parameters of the prepared nanoformulations.

\section{Drug entrapment}

Hydration of thin film of mor-nio was achieved using deionized distilled water in order to obtain drug-loaded niosomes. The hydration step is important for self-assembly of the surfactants and cholesterol as tiny polymeric vesicles entrapping morusin. ${ }^{61}$ Confirmation of entrapment efficiency was established using 
UV-Vis absorption spectroscopy. Fig. 2a depicts the absorbance of mor-nio in comparison with void nio and free morusin.

Morusin (free drug) shows $\lambda_{\max }$ at wavelength $269 \mathrm{~nm}$, where a major peak was observed as shown in (Fig. 2a). Same peak was observed in the case of mor-nio as well, depicting morusin entrapment in niosomes. However the peak was not as prominent as seen in the case of free drug which maybe due to the small amount of drug entrapment inside the niosomal nano carrier. The minor peak shifts observed could be due to the interaction of morusin with niosome constituents during synthesis and encapsulation processes. In case of void nio, no peak was observed. The entrapment efficiency of the particles was around $97 \pm 1.256 \%$ for every batch of particles synthesized with $70.55 \mu \mathrm{g}$ of the drug getting encapsulated (loading efficiency $-7.69 \pm 0.14 \%$ ) as seen in Table 1 . It has been widely reported that length of the alkyl chain of surfactants determines the diameter of vesicles. Vesicles obtained from stearyl $\left(\mathrm{C}_{18}\right)$ chain are known to yield larger particles with higher entrapment efficiencies, ${ }^{32}$ which can be seen from our results by the use of span 60 as the surfactant. For every batch of nanoparticles synthesized, the encapsulation, yield and loading efficiencies had consistent values. The prepared mor-nio was totally water-dispersible as compared to free morusin, which is insoluble in water.

\section{Surface characterization of niosomes}

In order to study the chemical nature of niosomes, we performed FTIR spectroscopy studies. In the study, we used void nio and free drug as controls for the comparative analysis of chemical bonding properties and peak shifts in our drug loaded nano formulation. FTIR measures the selective absorption of light by the vibration modes of specific chemical bonds in the sample. Vibrations arising due to the functional groups are involved in such interactions, which may alter frequency and intensity. ${ }^{51,61}$ Fig. 2b shows overlay of spectra from morusin, void nio and mor-nio. The small band around $2978 \mathrm{~cm}^{-1}$ is attributed to the $\mathrm{C}-\mathrm{H}$ stretching, which may be caused due to the $-\mathrm{CH},-\mathrm{CH}_{2}$ and $-\mathrm{CH}_{3}$ stretching vibrations characteristic of morusin. ${ }^{62}$ However in case of void nio and mor-nio the band is around $2913 \mathrm{~cm}^{-1}$. Moreover the signals around $1645 \mathrm{~cm}^{-1}$ and $1585 \mathrm{~cm}^{-1}$ can be observed in both drug and drug loaded niosomes while it's completely absent in void nio. Additionally,
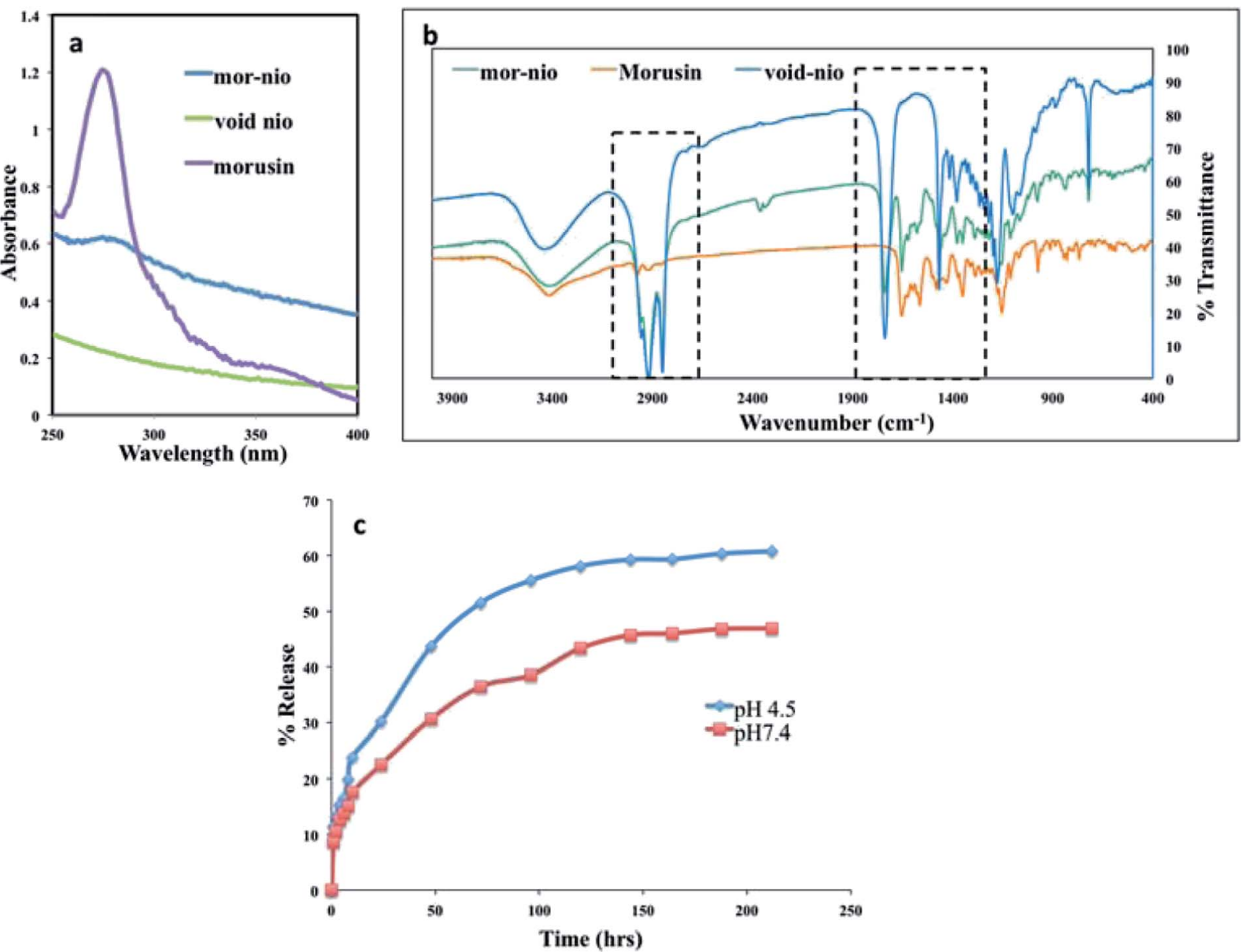

Fig. 2 (a) UV-absorption overlay spectra for free morusin, mor-nio and void nio. The characteristic peak for morusin is around 269 nm, which could be also observed in mor-nio. The void nio provided a flat profile (b) FT-IR spectra of mor-nio in comparison to native morusin and void nio as a control. (c) Sustained drug release profile for morusin nano formulation. An enhanced release was observed in acidic $\mathrm{pH}$ establishing mornio as an effective drug delivery system for $\mathrm{pH}$ dependent dug release in cancer cells. 
we could also see significant overlap in the spectra of void and mor-nio, which suggests that the chemical nature of the niosome remains unaltered. The FTIR analysis implies that there was no chemical interaction between the drug and the nanoparticle that could modify its chemical structure during the study and morusin was only physically encapsulated in the niosomes.

\section{In vitro drug release}

The drug release kinetics of mor-nio was studied using UV-Vis spectrophotometer for a period of 9 days under controlled conditions of $37{ }^{\circ} \mathrm{C}$ with constant stirring at $130 \mathrm{rpm}$ throughout the experiment. The most important prerequisite for a drug delivery vehicle is the slow and sustained release of drug. Percentage of morusin released from mor-nio at $\mathrm{pH} 4.5$ and $\mathrm{pH} 7.4$ under in vitro conditions can be observed in Fig. 2c. The drug release was biphasic with an initial burst release happening at the start, around $1^{\text {st }} \mathrm{h}(8.5 \%-\mathrm{pH} 7.4$ and $11.4 \%-$ $\mathrm{pH}$ 4.5) which can be attributed to the diffuse mechanism from outer layers of niosomes as well as minute surface-bound drug molecules. ${ }^{63}$ Morusin release from niosomes was perceived to be $\mathrm{pH}$ dependent, resulting in a slow drug release at physiological $\mathrm{pH} 7.4$ and a higher release at acidic $\mathrm{pH} 4.5(\mathrm{pH}$; depicting the acidic endosomal compartment after endocytosis), which is a prerequisite for transport of anticancer drugs in the acidic environment of cancer cells. Followed by burst release, a gradual release curve was observed after $24 \mathrm{~h}$ with $30.2 \%(21.32 \mu \mathrm{g})$ of drug being released till this point in acidic $\mathrm{pH}$. After $48 \mathrm{~h}$, the drug release increased to $43.6 \%(30.80 \mu \mathrm{g})$. At $120 \mathrm{~h}$, approximately $58.1 \%$ of morusin was released at acidic condition however at physiological $\mathrm{pH}$, only $43.3 \%$ was released following which there was a stationary phase. The niosome membrane ensures that the encapsulated drug crosses the membrane, which causes slower and delayed release. Also the amount of cholesterol included in the formulation affects the release kinetics, with high $\mathrm{CH}$ content causing slow release of the drug from the vesicle due to decreased permeability of the niosomal membrane but higher encapsulation efficiency that can be seen in case of our mor-loaded niosomes where the encapsulation efficiency was found to be around $97 \% .^{63}$

\section{Cytocompatibility analysis}

In order to determine whether the synthesized niosomes were non-toxic or could affect the growth of cells, we performed compatibility analysis in normal and cancer cells. Studies have proven niosomes to be safe without any cytotoxic effects. ${ }^{34,64,65}$ As could be seen from (Fig. 3), the cellular viability was above $80 \%$ in all the cell lines even at high concentration of particles $\left(1000 \mu \mathrm{g} \mathrm{ml}^{-1}\right)$. Thus, we confirm the particles synthesized were highly compatible to cells under study and could be utilized for further bio-applications without any modifications.

\section{Cellular uptake of mor-nio}

The uptake of niosomes by cancer cells was assessed using C-6 nio. Fig. 4 depicts the CLSM images of different cell lines treated with coumarin-6 loaded niosomes. The cells were incubated with $100 \mu \mathrm{g} \mathrm{ml} \mathrm{m}^{-1}$ of C-6 nio for 2 hours following which lysotracker and nuc blue were added in order to stain the acidic organelles and nucleus. The particles can be seen accumulated predominantly in the cytoplasm as evident from the green fluorescence of coumarin- 6 while controls show no fluorescence, thereby negating any auto fluorescence from the cells themselves.

HT-29 (Fig. 4b) cells had higher accumulation of particles as seen by strong green fluorescence compared to other cells. SKOV-3 (Fig. 4q) is also seen to have higher accumulation, which can be seen in the nucleus also however the cells were least susceptible to mor-nio treatment as seen from cytotoxicity studies. In PANC-1 (Fig. 4g) and MDA-MB-453 (Fig. 4l) the fluorescent signals were weak signifying lower accumulation of particles. However, MDA-MB-453 was fairly inhibited by mornio treatment as shown in cytotoxicity study. These observations indicate that lower particle accumulation was enough to exert significant toxicity to the cells.

Also, the images confirmed the endosome-mediated entry of particles as they can be readily seen accumulated in lysosomes where overlapping signals of nanoparticles and lysosomes could be visualized.

\section{Therapeutic efficacy of mor-nio}

The sustained toxic effect of the drug is stalled due to its poor bioavailability, a desired property for successful anticancer therapy. Therefore, to evaluate the therapeutic potential of mornio in comparison to free drug; we carried out dose and timedependent in vitro cell viability studies using alamar blue assay in HT-29, PANC-1, MDA-MB-453, SKOV-3 cells and L929 cells. Cells were subjected to five different concentrations $(50 \mu \mathrm{g}$ $\mathrm{ml}^{-1}, 100 \mu \mathrm{g} \mathrm{ml}^{-1}, 250 \mu \mathrm{g} \mathrm{ml}^{-1}, 500 \mu \mathrm{g} \mathrm{ml}^{-1}, 1000 \mu \mathrm{g} \mathrm{ml}^{-1}$ ) of mor-nio and free morusin for 6, 24 and 48 hours. In cancer cell lines, we could perceive instant cell death and reduced cell viability within 24 hours when treated with mor-nio (Fig. 5). However, at the lowest dose (50-100 $\left.\mu \mathrm{g} \mathrm{ml}^{-1}\right)$, the effect was comparatively less pronounced while maximum cytotoxic effect was observed at $1000 \mu \mathrm{g} \mathrm{ml}^{-1}$ concentration after $48 \mathrm{~h}$. This could be due to the fact that mor-nio were able to reach a therapeutic concentration after 48 hours to impart cytotoxic effects to cancer cells. Toxicity of free drug was performed keeping in mind the amount of drug encapsulated in $1000 \mu \mathrm{g} \mathrm{ml}^{-1}$ of mornio i.e. $70.55 \mu \mathrm{g}$, which was calculated using eqn (2). Compared to mor-nio, the free drug displayed less cytotoxicity towards cancer cells which maybe due to its hydrophobicity indicating the advantage of encapsulating the drug inside niosomal carrier. In case of free morusin, the cell viability was - HT29 (48.4\%). PANC-1 (62.77\%), MDA-MB-453 (53.02\%), SKOV-3 $(62.84 \%)$ after $24 \mathrm{~h}$ at $70.55 \mu \mathrm{g} \mathrm{m}{ }^{-1}$ concentration (which is the amount of drug entrapped in $1000 \mu \mathrm{g} \mathrm{ml^{-1 }}$ of mor-nio) however a decline in viability was seen after $48 \mathrm{~h}$. On the other hand, mor-nio decreased the cell viability to a significant level after $24 \mathrm{~h}$ at $1000 \mu \mathrm{g} \mathrm{ml}^{-1}$ compared to the free drug -HT29 (33.05\%), PANC-1 (46.13\%), MDA-MB-453 (39.93\%), SKOV-3 (59.17\%). As seen in drug release (Fig. 2c) at $24 \mathrm{~h}, 30.23 \%$ $(21.32 \mu \mathrm{g})$ was released in acidic environment while it increased 
a

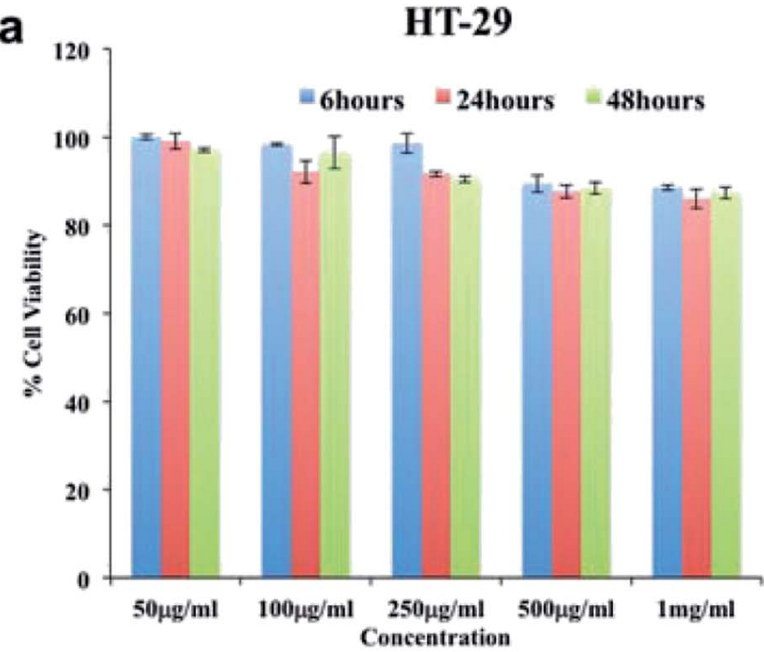

C

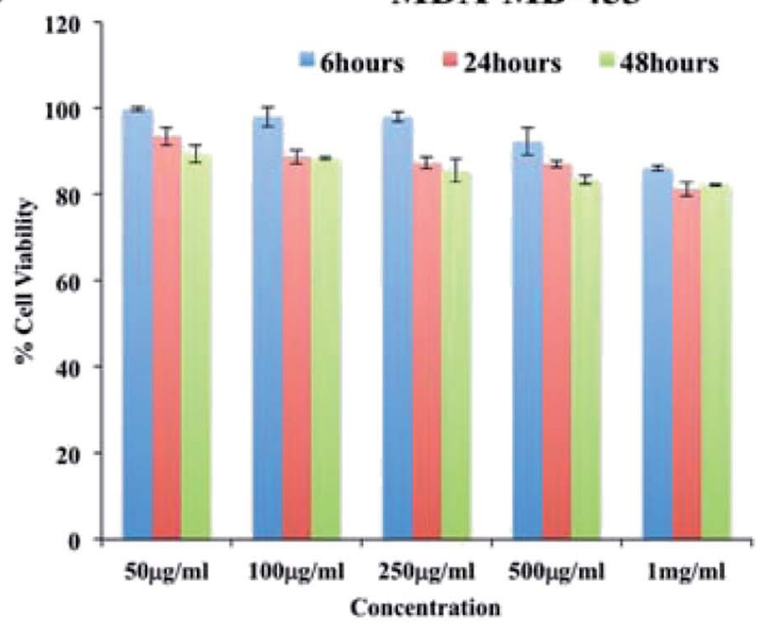

b

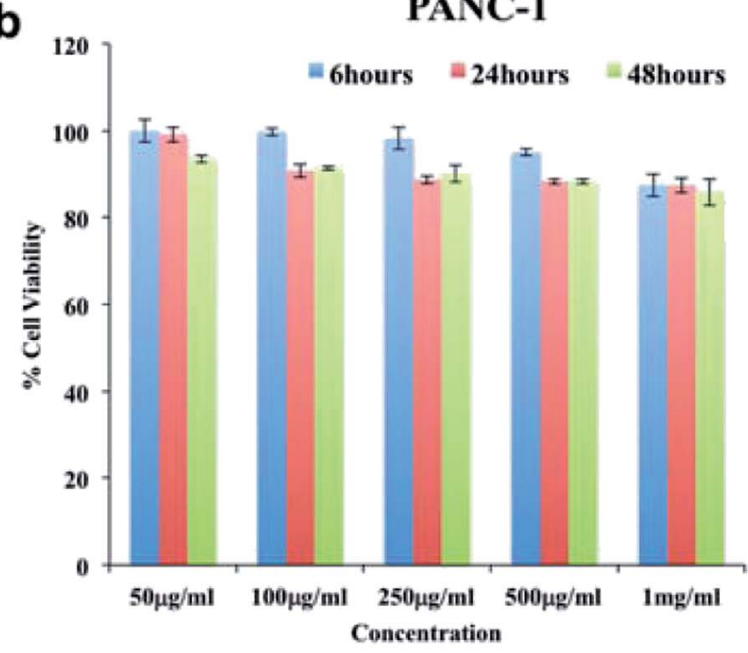

d

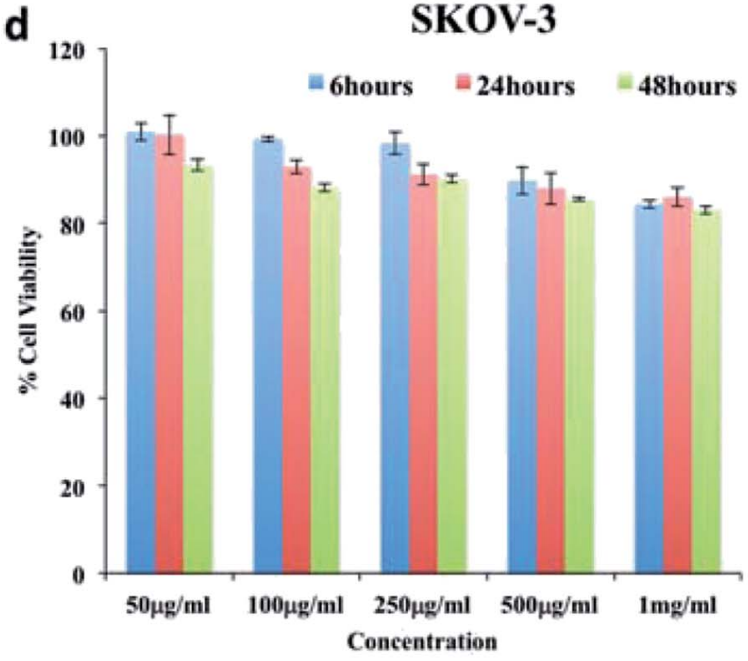

\section{L929}

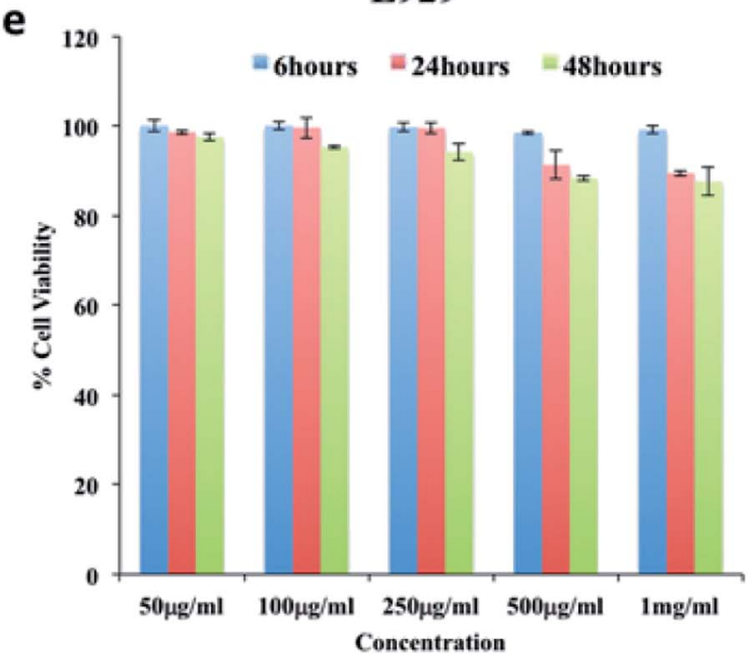

Fig. 3 Cytocompatibility of void niosomes tested against cancer (a) HT-29, (b) PANC-1, (c) MDA-MB-453 (d) SKOV-3, cells and normal cell line (e) L929. The particles were highly cytocompatible as depicted by high viability in all cell lines even at highest concentration of particles used.

to $43.66 \%(30.80 \mu \mathrm{g})$ after $48 \mathrm{~h}$, thus correlating with the cytotoxicity data with an increase in cell death at $48 \mathrm{~h}$ due to augmented release of the drug. The viability after $48 \mathrm{~h}$ in case of morusin niosomes was - HT29 (26.32\%). PANC-1 (35.88\%), MDA-MB-453 (21.53\%), SKOV-3 (46.3\%) whereas in case of free drug it was - HT29 (35.21\%), PANC-1 (55.68\%), MDA-MB-453 


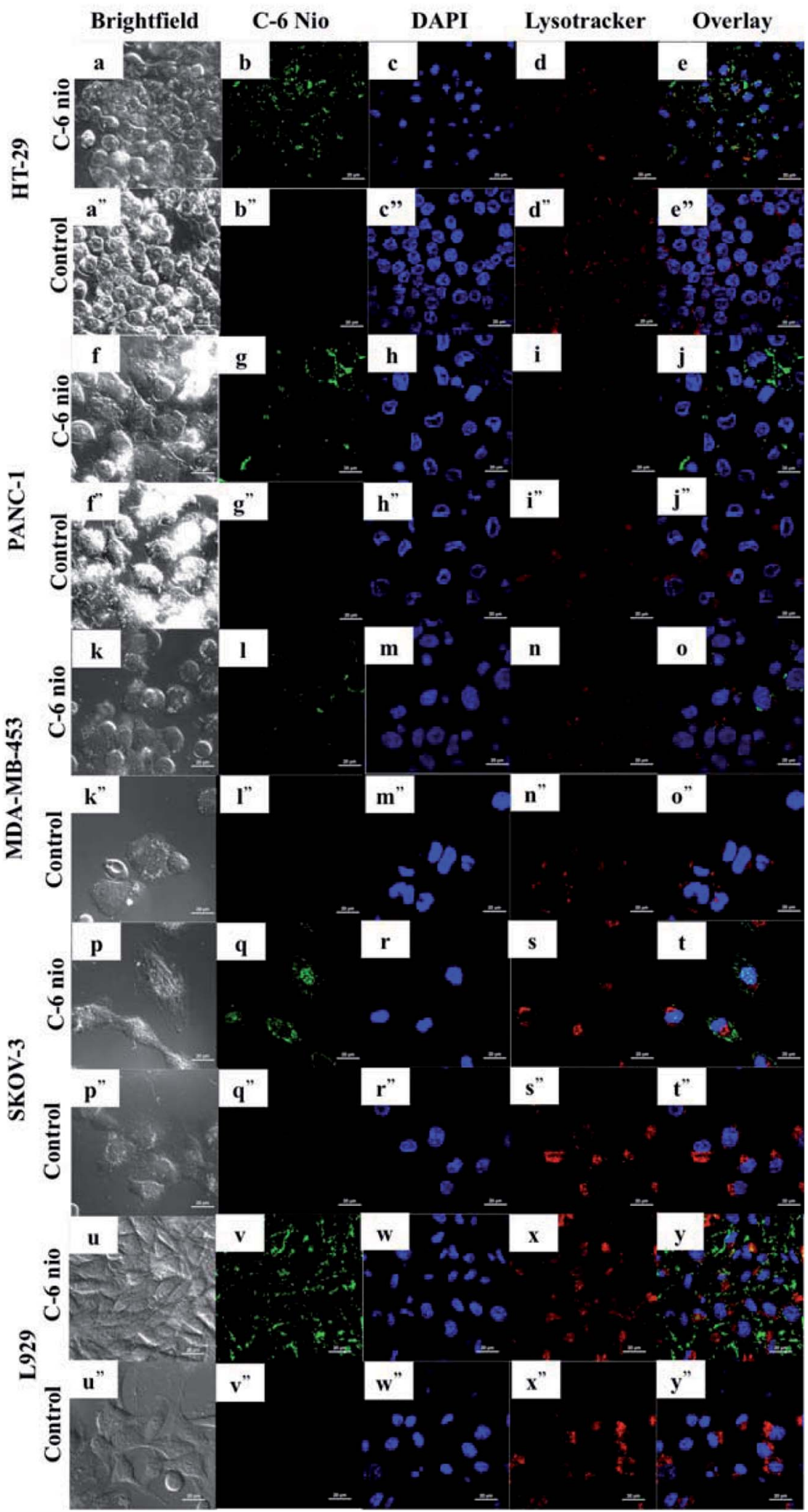

Fig. 4 Confocal microscopy images demonstrating cellular internalization of coumarin- 6 niosomes by cancer cells. The green fluorescent intensity from cells with C-6 nio was clearly evident, signifying the enhanced uptake of niosomes by cancer cells. Nuclei were stained with NucBlue and lysosomes with lysotracker deep red to show nanoparticles co-localization. Scale bars represent $20 \mu \mathrm{m}$. 
MOR-NIO
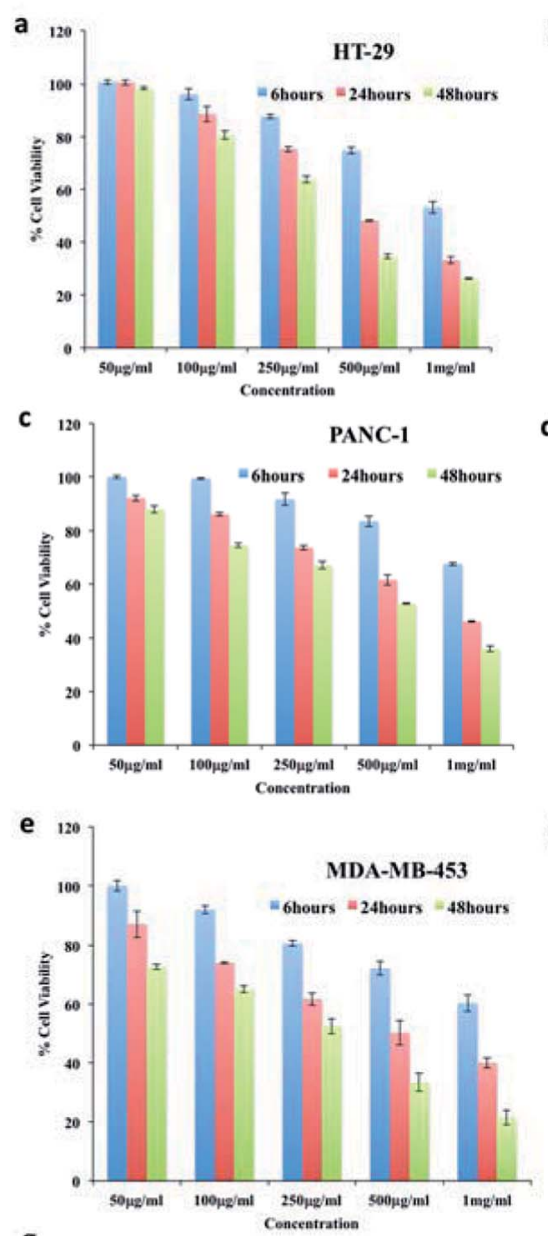

g
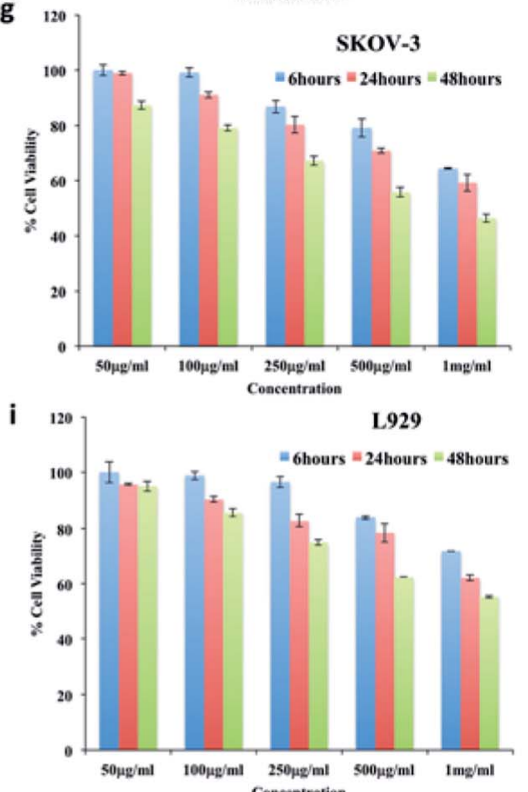

FREE MORUSIN
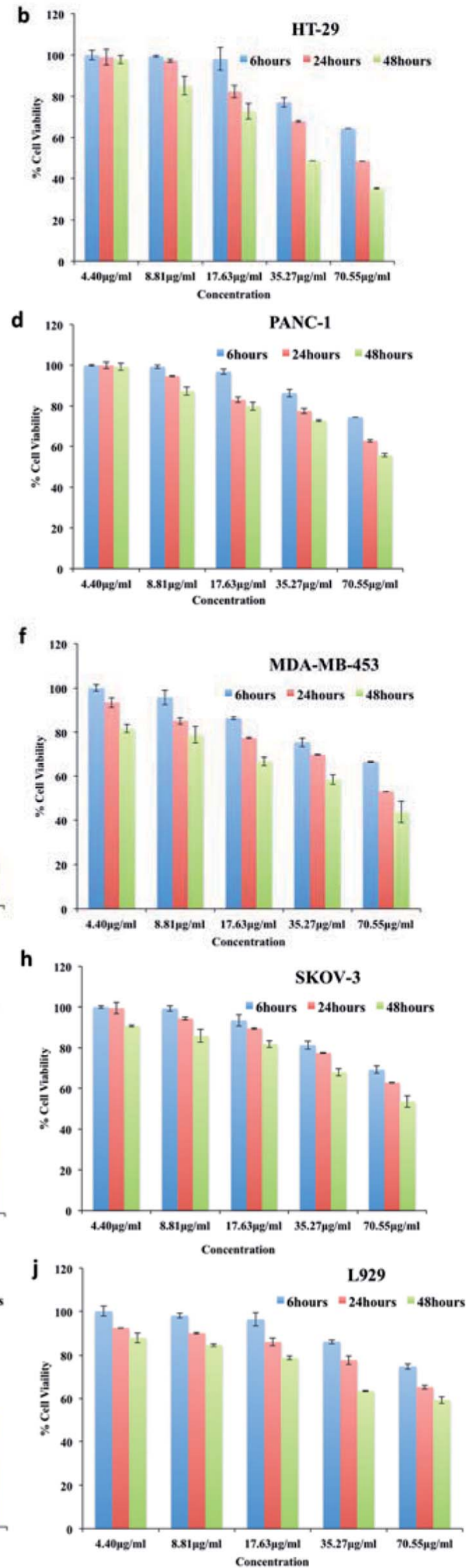

Fig. 5 Therapeutic efficacy of morusin-loaded niosomes and free morusin towards (a and b) HT-29 (c and d) PANC-1 (e and f) MDA-MB-453 (g and h) SKOV-3 and (i and j) L929 cell line. Compared to the free drug, more inhibition was observed in the case of morusin-loaded niosomes in all cell lines. 
(43.77\%), SKOV-3 (53.55\%). Though, similar particle accumulation was observed in case of L929 cells as well (Fig. 4v), viability remained at approximately $80 \%$ (Fig. $5 \mathrm{i}$ and $\mathrm{j}$ ). The reason for this could be the high mitotic index (cell doubling rate) of L929 compared to other cell lines which could have limited the effect of the drug when compared to rapidly dividing cells.

HT-29 was seen to be the most susceptible cell line to mornio treatment. NF- $\kappa \mathrm{B}$ activation is known to promote tumor progression in colorectal cancers. Lee et al. ${ }^{20}$ reported that morusin was able to significantly suppress constitutive activity of NF- $\kappa$ B via classical pathway. Dephosphorylation of Akt, PDK1 and P13 was also observed along with activation of caspase 9 due to downregulation of XIAP expression, caspase-8, and -3 due to morusin treatment in colorectal cancer cells. Thus, taking into account all the above factors, multiple pathways are known to be involved in inducing apoptosis in HT-29 cells by morusin which may be the reason of enhanced inhibition observed by mor-nio treatment. In case of pancreatic cancer, constitutive activation of STAT 3 has been reported causing tumor cell survival, proliferation and metastasis. Inhibition of STAT-3 and downregulation of its regulated gene products-IAP1, IAP2, Bcl-2, survivin, cyclin, COX-2, VEGF, MMP-9 was seen in pancreatic tumor cells in parallel with inhibition of JAK1, JAK2 upon morusin treatment leading to apoptosis. ${ }^{22}$

Triple negative breast cancers (TNBCs) are cancers that do not express progesterone receptor (PR), estrogen receptor (ER), and the HER2 isoform of epidermal growth factor receptor (EGFR) and hence due to lack of a specific therapeutic target triple negative breast cancers have limited treatment options. ${ }^{66}$ Mor-nio was seen to significantly reduce the cell viability of MDA-MB-453, a triple negative breast cancer line upon treatment. Morusin has been shown to increase expression of pro-apoptotic marker protein Bax, reduce antiapoptotic protein survivin as well as cause cleavage of caspase 9, -3 and PARP in a dose and time dependent manner in triple negative breast cancer cell lines indicating its ability at targeting anti and pro-apoptotic signals that may increase anti-cancer activity. ${ }^{19}$ The mechanism of action however remains elusive and its known that STAT3 inhibition affects expression of downstream targets-Bax and survivin, so maybe morusin works by inhibiting STAT 3 also. Despite inducing apoptosis in breast cancer cells, apoptotic rate was low compared with cytotoxicity rate for same concentration of morusin which means morusin may induce other types of cell death like necrosis and autophagy in breast cancer cells. ${ }^{19}$ Thus, our synthesized mor-nio were able to deliver the drug into cancer cells by enhanced permeation and retention (EPR) effect for maximizing the therapeutic effects of morusin even in a drug resistant cell line and cause its inhibition while reducing systemic toxicity. A high level of receptor expressionEGFR, insulin growth factor receptor-1 (IGF-1R), folate, CXCR4 has been reported in TNBC tissues ${ }^{66}$ which may also be the mode of action by which morusin mediated toxicity in MDAMB-453, however this remains to be confirmed by further studies.
Epithelial ovarian cancer (EOC), remains the leading cause of death among gynecological malignant tumors. ${ }^{67}$ Chemoresistance and treatment failure often occurs due to cellular resistance to apoptosis, thereby compounds, which may treat the tumor other than by inducing apoptosis, may provide a better insight in treating EOC. Recently, morusin was shown to kill ovarian cells by paraptosis rather than inducing apoptosis. ${ }^{67}$ It was also shown to inhibit colony formation in EOC cells occurring due to the dual effect of cell death and cell proliferation inhibition. However, caspase -3 nor -9 were found to be inactivated by morusin in EOC ruling out apoptosis mediated death. In our study, though significant toxicity was observed, SKOV-3 cells were the most resistant of the cell lines under study. Thus, it is presumed that morusin can activate diverse cell death pathways depending on different cancer cell types, which explains our cytotoxicity data, with sensitivity of different cancer types to morusin inhibition.

The enhancement of cell death within $24 \mathrm{~h}$ treatment of morusin niosomes compared to free morusin may be due to the advantage of nanoparticulate mode of drug delivery. Also it has been seen that use of span 60 as a surfactant has been known to increase the anti-tumor action of the drug. ${ }^{68}$ Since nanoscale particles are easy to internalize within cells, increased drug retention could be achieved. The cytotoxicity data implies that mor-nio can competitively demonstrate excellent anticancerous effect than free morusin.

\section{Live/dead viability assay}

In order to analyze activation of apoptosis in cancer cells when treated with morusin niosomes, we performed live/dead viability assay to visualize early apoptotic and necrotic cells. The viability of cells was analyzed post $24 \mathrm{~h}$ using live dead viability staining. EthD-1 enters dead cells via the damaged membrane, binds to their nuclei, and releases red fluorescence while live cells enzymatically convert calcein AM in fluorescent calcein which emits green fluorescence. $500 \mu \mathrm{g} \mathrm{ml}{ }^{-1}$ concentration was sufficient to elicit cytotoxicity to cancer cells as confirmed by cytotoxicity analysis therefore the same concentration was utilized for this assay. Significant number of dead cells could be observed with morusin loaded niosome treated groups compared to free drug at the same concentration (Fig. 6).

In case of HT-29 and MDA-MB-453, when cells were treated with free morusin, a large number of viable cell population (Fig. $6 b^{\prime}$ and $j^{\prime}$ ) could be observed with some necrotic cells (Fig. $6 \mathrm{c}^{\prime}$ and $\mathrm{k}^{\prime}$ ) as well. Compared to free morusin, significant amount of dead cells were observed when cells were treated with mor-nio (Fig. 6c* and $\mathrm{k}^{*}$ ) as established by enhanced fluorescent intensity of Etbr (red). Similar effects could be observed in PANC-1- (Fig. 6 6*), however the fluorescent intensity of Etbr was comparatively less compared to HT-29 and MDA-MB-453 which can be confirmed by cytotoxicity analysis wherein at $500 \mu \mathrm{g} \mathrm{ml}^{-1}$ concentration, cell viability for mor-nio was $48.1 \%$ for HT-29 and $50.17 \%$ for MDA-MB-453 while for PANC-1 it was $61.65 \%$ after 24 h. However, in case of SKOV-3, both morusin (Fig. 6o') and mor-nio (Fig. 60*) had similar effect on the cells but the red fluorescence coming from the dead cells was more pronounced 
HT-29

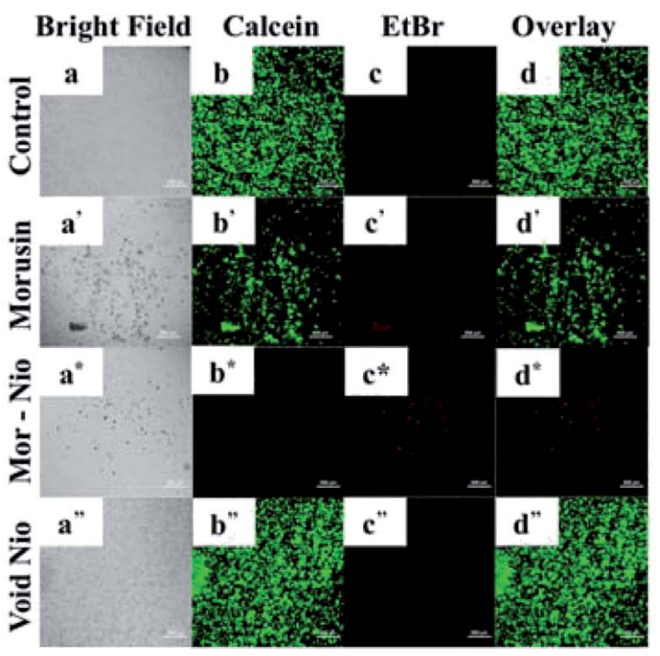

MDA-MB-453

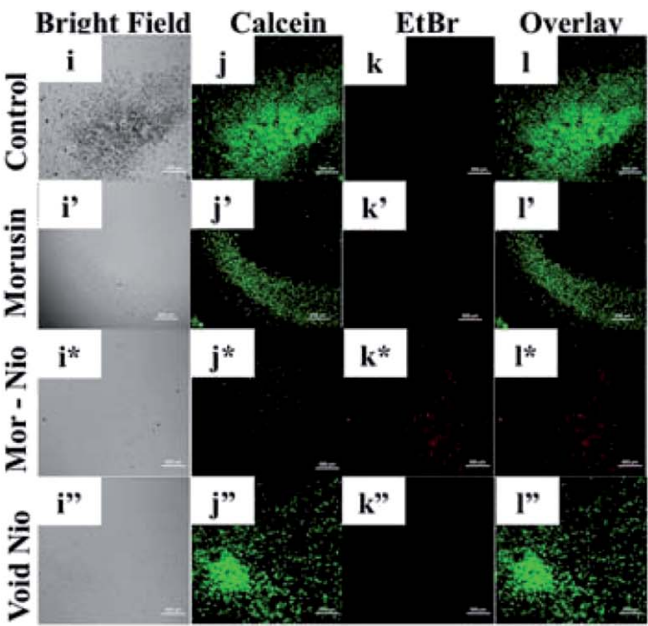

PANC-1

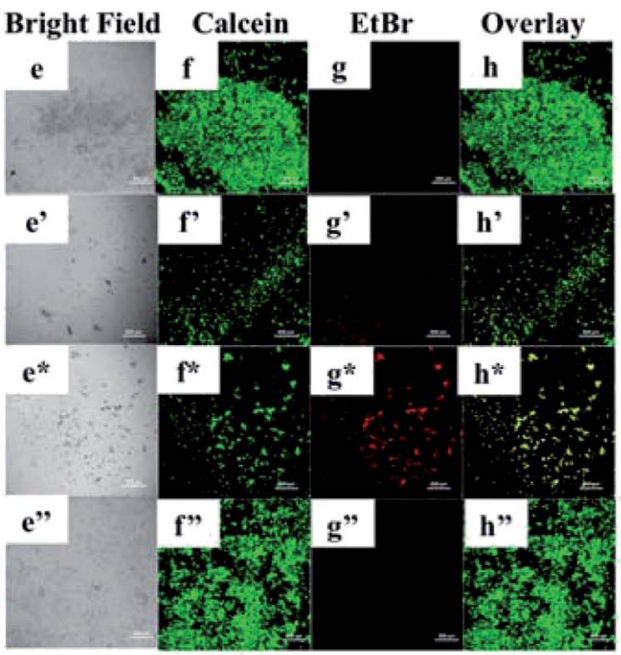

SKOV-3

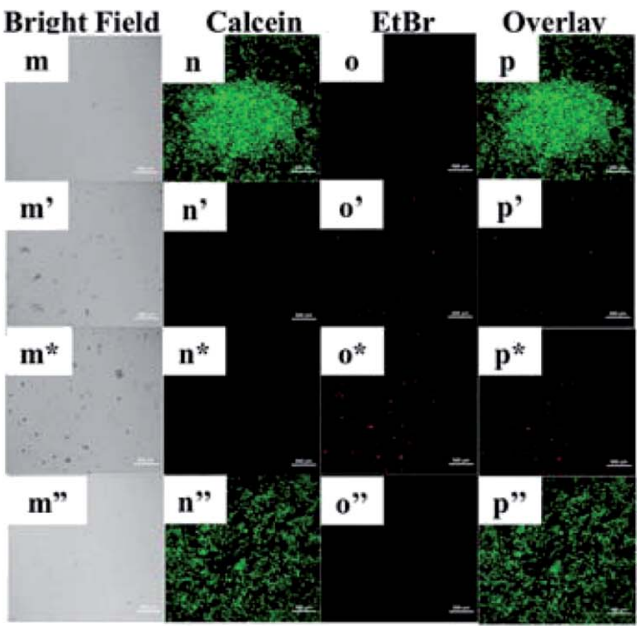

L929

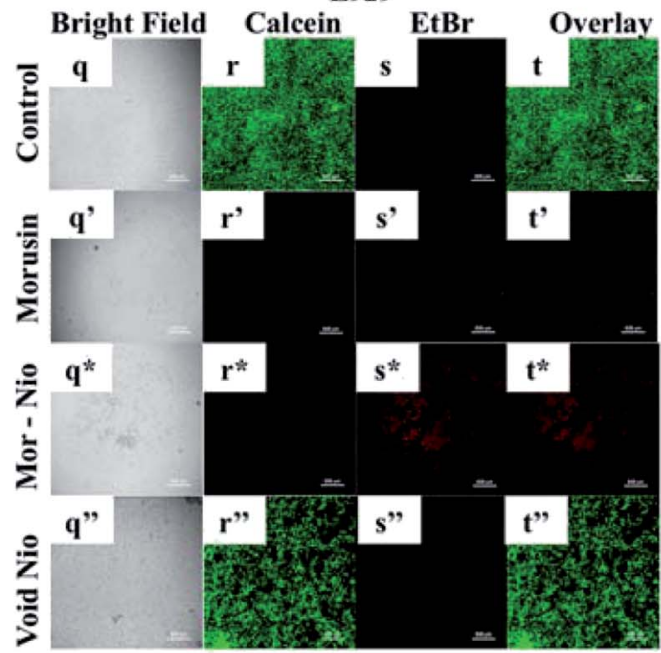

Fig. 6 Therapeutic efficacy of morusin loaded, free morusin and void niosomes against cancer cell lines using Live/Dead Assay. The control cells and cells treated with void niosomes were live as evident by the green fluorescence. The cells treated with morusin niosomes showed considerable toxicity with minimal number of live cells and increased amount of dead cells (red fluorescence) compared to free morusin where there were both viable and dead cell populations. Scale bars represent $500 \mu \mathrm{m}$. 


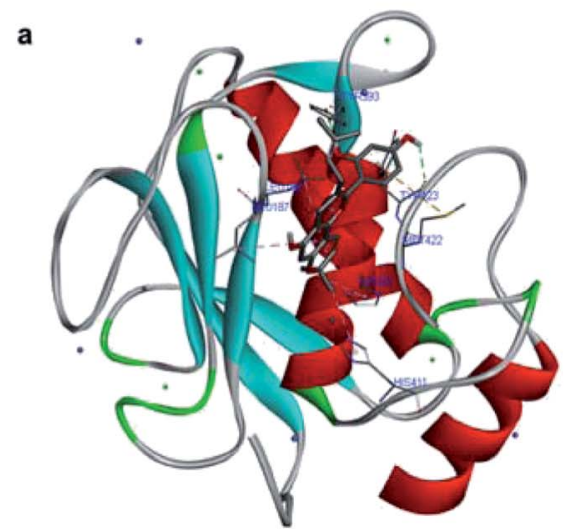

Morusin Binding Pocket b

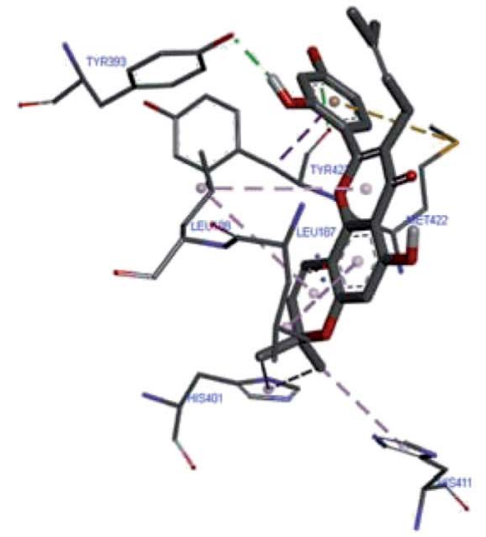

Amino acid residues of MMP-9 interacting with Morusin

\begin{tabular}{|l|c|c|c|}
\hline hydrogen bonds & cation-pi & hydrophobic & other \\
\hline TYR393 (-0.6454) & TYR423 (-1.8018) & LEU187 (-0.8944) & LEU188 (-1.0581) \\
\hline & & MET422 (-0.6481) & ASP185 (-0.4799) \\
\hline & & HIS401 (-0.2661) & HIS411 (-0.217) \\
\hline & & & GLU402 (-0.096) \\
\hline
\end{tabular}

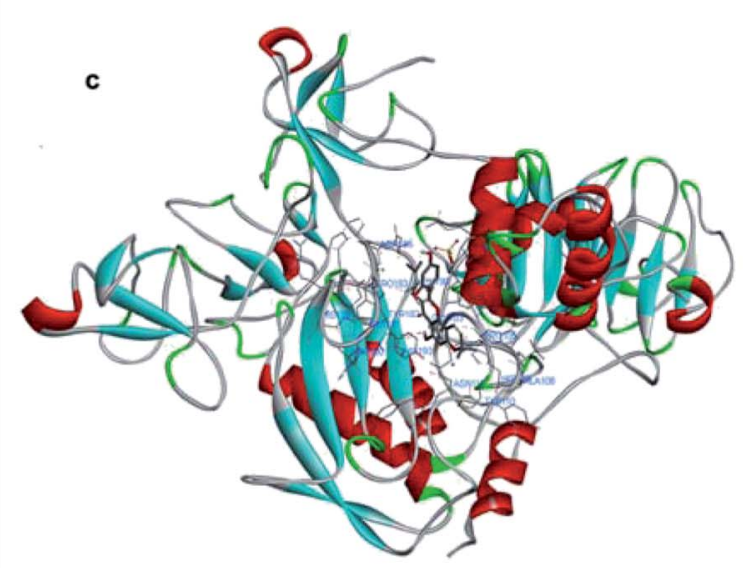

Morusin Binding Pocket d

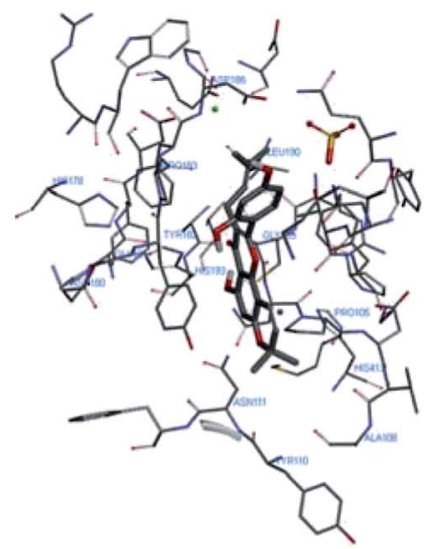

Amino acid residues of MMP-2 interacting with Morusin

\begin{tabular}{|l|l|l|l|}
\hline \multicolumn{1}{|c|}{ polar } & \multicolumn{1}{c|}{ cation-pi } & \multicolumn{1}{c|}{ hydrophobic } & \multicolumn{1}{c|}{ other } \\
\hline ASN111 (-1.0066) & TYR182 (-2.8653) & PRO105 (-0.8123) & THR56 (-1.6159) \\
\hline ASN55 (-0.9011) & & LEU190 (-0.516) & PRO183 (-0.8415) \\
\hline HIS193 (-0.4709) & & & ASP185 (-0.2221) \\
\hline
\end{tabular}

Fig. 7 Molecular docking studies showing interaction between the metalloproteinases (MMP-9 and MMP-2) and morusin. (a and b) It shows the amino acid residues of MMP-9 interacting with morusin ( $c$ and d) Probable binding pocket for morusin on MMP-2 and its interacting residues, which help in attaining stable conformational state via polar and non-polar interactions. 
in the case of mor-nio as inferred from cytotoxicity data where cell viability after $24 \mathrm{~h}$ was $77.45 \%$ when free drug was used and $70.78 \%$ when cells were treated with mor-nio. Both control cells (Fig. 6d, h, l and p) and cells that were treated with void nio (Fig. $6 \mathrm{~d}^{\prime \prime}, \mathrm{h}^{\prime \prime}, \mathrm{l}^{\prime \prime}$ and $\mathrm{p}^{\prime \prime}$ ) showed higher percentage of live cells as evident by high green fluorescent intensity thus indicative of the healthy state of cells and establishing that cell death was induced by the drug and not by the niosome nano-carrier. The results provide visual quantitative support to the cytotoxicity observations that mor-nio could induce cytotoxic effects in cancer cells.

\section{Docking studies}

It has been reported by a number of studies that morusin exerts its inhibitory effect on cancer cells by inhibiting one or two key molecules, which are required for cancer pathogenesis like STAT- 3 and NF- $\kappa$ B pathways. The inhibition of the matrix metalloproteinases gelatinases (MMP-2 and MMP-9) by morusin has also been described in literature. ${ }^{\mathbf{2 0 , 2 4 , 2 5}}$ Also, there is an elevation of these MMPs in colorectal cancer, pancreatic cancer, ovarian cancer and breast cancer as discussed previously. Thus, in order to check whether there was any interaction between morusin and MMPs, we performed molecular docking studies using Autodock tool. The study showed that the MMPs interaction with morusin was influenced by hydrogen bonding with active site residues. We performed blind docking study to get an unbiased estimate of the binding site for morusin on MMPs. The Gibbs free binding energy $(\Delta G)$ of the MMP-9 morusin complex was $\Delta G=-7.35 \mathrm{~kJ} \mathrm{~mol}^{-1}$ with inhibitory constant $\left(K_{\mathrm{i}}\right)$ value of $4.09 \mu \mathrm{M}$. Whereas, for MMP-2 morusin complex $\Delta G=$ $-8.33 \mathrm{~kJ} \mathrm{~mol}^{-1}$ with $K_{\mathrm{i}}$ value of $786.78 \mathrm{nM}$. In case of MMP-9morusin complex, there was H-bonding of Tyr 393 with morusin while Tyr 423 was engaged in cationic-pi interactions. Leu 187, Met 422 and His 401 provided the hydrophobic interactions. There was no interaction between morusin and $\mathrm{Zn}$ of MMPs (Fig. 7a and b). In case of MMP-2, active site amino acids Asn 111, Asn 55 and His 193 were engaged in polar contact with morusin, while Tyr 182 was found to have cation-pi interactions. Pro 105 and Leu 190 were the two amino acid residues responsible for the hydrophobic interactions between the MMP2-morusin complexes (Fig. 7c and d). This is the first computational study to show the probable binding interaction site for morusin on MMPs.

\section{Conclusion \& future perspectives}

The present research work details the development of morusin loaded niosomal nano-formulation for cancer inhibition. The therapeutic efficacy was imparted by morusin, which is a potent naturally derived chemotherapeutic agent as confirmed by the results presented in this report. The particles, with an average size of $479 \mathrm{~nm}$, displayed high drug loading and encapsulation efficiencies, in addition to rendering aqueous dispersibility to the usually hydrophobic morusin. A steady and sustained drug release could be observed for morusin from the niosomes along-with increased release in acidic $\mathrm{pH}$ thus not only proposing its efficient release in the acidic environment of cancer cells, but additionally reducing the spontaneous drug release under physiological $\mathrm{pH}$ of normal cells. The toxicity of morusin niosomes was tested against 4 cancer cell lines of different lineages to assess the degree of toxicity depending on the cell lineage. According to our study, the cancer cells susceptibility to mor-nio treatment was in the following order: HT-29>MDA-MB-453>PANC-1>SKOV-3. Our findings suggest that, regardless of the cell type or origin, all the cells under investigation were highly susceptible to the nano formulation in a concentration dependent manner, predicting the utilization of this morusin-niosomal nanoformulation for multiple types of cancers. Morusin-nio was able to inhibit the survival of MDAMB-453, which is a highly drug resistant triple negative breast cancer cell line. We also performed docking studies to analyze probable binding of morusin with MMPs. We anticipate that morusin loaded niosomes will open new scenarios for precise delivery of morusin to cancer sites as well as lay foundation for the development of novel targeted therapies in future.

\section{Conflicts of interest}

There are no conflicts to declare.

\section{Acknowledgements}

Srishti Agarwal would like to acknowledge her sincere gratitude to the Ministry of Education, Culture, Sports, Science and Technology (MEXT), Japan for the financial support under the Monbukagakusho fellowship during the research. S. A. would also like to express her sincere gratitude to the Inoue Enryo Research Grant (Toyo University).

\section{References}

1 G. W. Prager, S. Braga, B. Bystricky, C. Qvortrup, C. Criscitiello, E. Esin, et al., ESMO Open, 2018, 3(2), e000285.

2 J. Xie, Z. Fan, Y. Li, Y. Zhang, F. Yu, G. Su, L. Xie and Z. Hou, Int. J. Nanomed., 2018, 13, 1381-1398.

3 Y. Davatgaran-Taghipour, S. Masoomzadeh, M. H. Farzaei, R. Bahramsoltani, Z. Karimi-Soureh, R. Rahimi and M. Abdollahi, Int. J. Nanomed., 2017, 12, 2689-2702.

4 S. K. Singh, J. W. Lillard Jr. and R. Singh, Cancer Lett., 2018, 427, 49-62.

5 R. Kamal, V. D. Chadha and D. K. Dhawan, Nanomedicine, 2018, 14, 1059-1071.

6 F. Aghapour, A. A. Moghadamnia, A. Nicolini, S. N. M. Kani, L. Barari, P. Morakabati, L. Rezazadeh and S. Kazemi, Biochem. Biophys. Res. Commun., 2018, 500, 860-865.

7 A. Patra, S. Satpathy, A. K. Shenoy, J. A. Bush, M. Kazi and M. D. Hussain, Int. J. Nanomed., 2018, 13, 2869-2881.

8 R. D. Nisha Tyagi, J. Begun and A. Popat, Int. J. Pharm., 2017, 518.

9 K. Liang, J. E. Chung, S. J. Gao, N. Yongvongsoontorn and M. Kurisawa, Adv. Mater., 2018, 30, e1706963.

10 C. Basmadjian, Q. Zhao, E. Bentouhami, A. Djehal, C. G. Nebigil, R. A. Johnson, M. Serova, A. de Gramont, 
S. Faivre, E. Raymond and L. G. Desaubry, Front. Chem., 2014, 2, 20.

11 J. Li, S. G. Kim and J. Blenis, Cell Metab., 2014, 19, 373-379. 12 L. Reddy, B. Odhav and K. D. Bhoola, Pharmacol. Ther., 2003, 99, 1-13.

13 C. Geng, S. Yao, D. Xue, A. Zuo, X. Zhang, Z. Jiang, Y. Ma and J. Chen, Zhongguo Zhongyao Zazhi, 2010, 35, 1560-1565.

14 H. J. Lee, H. Lyu da, U. Koo, K. W. Nam, S. S. Hong, K. O. Kim, K. H. Kim, D. Lee and W. Mar, Arch. Pharmacal Res., 2012, 35, 163-170.

15 H. H. Ko, J. J. Wang, H. C. Lin, J. P. Wang and C. N. Lin, Biochim. Biophys. Acta, 1999, 1428, 293-299.

16 A. M. Abd El-Mawla, K. M. Mohamed and A. M. Mostafa, Sci. Pharm., 2011, 79, 951-961.

17 Z. G. Yang, K. Matsuzaki, S. Takamatsu and S. Kitanaka, Molecules, 2011, 16, 6010-6022.

18 H. Li, Q. Wang, L. Dong, C. Liu, Z. Sun, L. Gao and X. Wang, J. Exp. Clin. Cancer Res., 2015, 34, 137.

19 S. Kang, E. O. Kim, S. H. Kim, J. H. Lee, K. S. Ahn, M. Yun and S. G. Lee, Oncol. Lett., 2017, 13, 4558-4562.

20 J. C. Lee, S. J. Won, C. L. Chao, F. L. Wu, H. S. Liu, P. Ling, C. N. Lin and C. L. Su, Biochem. Biophys. Res. Commun., 2008, 372, 236-242.

21 F. Wang, D. Zhang, J. Mao, X. X. Ke, R. Zhang, C. Yin, N. Gao and H. Cui, Oncotarget, 2017, 8, 57187-57200.

22 C. Kim, J. H. Kim, E. Y. Oh, D. Nam, S. G. Lee, J. Lee, S. H. Kim, B. S. Shim and K. S. Ahn, Pancreas, 2016, 45, 409-419.

23 S. L. Lim, S. Y. Park, S. Kang, D. Park, S. H. Kim, J. Y. Um, H. J. Jang, J. H. Lee, C. H. Jeong, J. H. Jang, K. S. Ahn and S. G. Lee, Am. J. Cancer Res., 2015, 5, 289-299.

24 W. L. Lin, D. Y. Lai, Y. J. Lee, N. F. Chen and T. H. Tseng, Toxicol. Lett., 2015, 232, 490-498.

25 Z. Vochyanova, M. Pokorna, D. Rotrekl, V. Smekal, P. Fictum, P. Suchy, J. Gajdziok, K. Smejkal and J. Hosek, PLoS One, 2017, 12, e0182464.

26 S. Parveen, R. Misra and S. K. Sahoo, Nanomedicine, 2012, 8, 147-166.

27 M. Orunoglu, A. Kaffashi, S. B. Pehlivan, S. Sahin, F. Soylemezoglu, K. K. Oguz and M. Mut, Mater. Sci. Eng., C, 2017, 78, 32-38.

28 Sauraj, S. U. Kumar, V. Kumar, R. Priyadarshi, P. Gopinath and Y. S. Negi, Carbohydr. Polym., 2018, 188, 252-259.

29 J. A. Loureiro, S. Andrade, A. Duarte, A. R. Neves, J. F. Queiroz, C. Nunes, E. Sevin, L. Fenart, F. Gosselet, M. A. Coelho and M. C. Pereira, Molecules, 2017, 22, 277.

30 M. Singh, P. Bhatnagar, S. Mishra, P. Kumar, Y. Shukla and K. C. Gupta, Int. J. Nanomed., 2015, 10, 6789-6809.

31 S. Bansal, G. Aggarwal, P. Chandel and S. L. Harikumar, J. Pharm. BioAllied Sci., 2013, 5, 318-325.

32 P. W. Aranya Manosroi, J. Manosroi, H. Sakai, F. Sugawara, M. Yuasa and M. Abe, Colloids Surf., B, 2003, 30, 129-138.

33 P. G. P. Kumar, Acta Pharm. Sin. B, 2011, 1, 208-219.

34 S. Taymouri and J. Varshosaz, Adv. Biomed. Res., 2016, 5, 48. 35 M. I. Khan, M. Asadullah, A. Saeed, M. A. Mahmood, M. Rehman and M. Ashfaq, Braz. J. Pharm. Sci., 2015, 51, 607-615.
36 S. Mandal, C. Banerjee, S. Ghosh, J. Kuchlyan and N. Sarkar, J. Phys. Chem. B, 2013, 117, 6957-6968.

37 N. Thakur Gurjeet Singh, in Nanobiomaterials in Galenic Formulations and Cosmetics, William Andrew Applied Science Publishers, 2016, ch. 7, vol. 10, pp. 149-174.

38 P. Arunothayanun, J. A. Turton, I. F. Uchegbu and A. T. Florence, J. Pharm. Sci., 1999, 88, 34-38.

39 A. Namdeo and N. K. Jain, J. Microencapsulation, 1999, 16, 731-740.

40 I. F. Uchegbu, J. A. Double, J. A. Turton and A. T. Florence, Pharm. Res., 1995, 12, 1019-1024.

41 L. Kanaani, I. Javadi, M. Ebrahimifar, H. Ebrahimi Shahmabadi, A. Akbarzadeh Khiyav and T. Mehrdiba, Asian Pac. J. Cancer Prev., 2017, 18, 365-368.

42 V. Sharma, S. Anandhakumar and M. Sasidharan, Mater. Sci. Eng., C, 2015, 56, 393-400.

43 Z. S. Bayindir and N. Yuksel, J. Pharm. Sci., 2010, 99, 20492060.

44 K. Jakubowska, A. Pryczynicz, J. Januszewska, I. Sidorkiewicz, A. Kemona, A. Niewinski, L. Lewczuk, B. Kedra and K. Guzinska-Ustymowicz, Dis. Markers, 2016, 2016, 9895721.

45 A. H. Said, J. P. Raufman and G. Xie, Cancers, 2014, 6, 366375.

46 M. W. Roomi, J. C. Monterrey, T. Kalinovsky, M. Rath and A. Niedzwiecki, Oncol. Rep., 2010, 23, 605-614.

47 X. Hu, D. Li, W. Zhang, J. Zhou, B. Tang and L. Li, Arch. Gynecol. Obstet., 2012, 286, 1537-1543.

48 H. Li, Z. Qiu, F. Li and C. Wang, Oncol. Lett., 2017, 14, 58655870.

49 K. D. Fagan-Solis, S. S. Schneider, B. T. Pentecost, B. A. Bentley, C. N. Otis, J. F. Gierthy and K. F. Arcaro, J. Cell. Biochem., 2013, 114, 1385-1394.

50 V. P. Ankita Borah, A. R. Girija, S. Balasubramanian, A. K. Rochani, T. Maekawa and D. Sakthi Kumar, NanoWorld J., 2017, 3(1), 1-10.

51 A. K. Rochani, S. Balasubramanian, A. Ravindran Girija, S. Raveendran, A. Borah, Y. Nagaoka, Y. Nakajima, T. Maekawa and D. S. Kumar, Int. J. Pharm., 2016, 511, 648-658.

52 A. R. Sivakumar B, Y. Nagaoka, S. Iwai, K. Venugopal, et al., RSC Adv., 2013, 3, 20579-20598.

53 H. Abdelkader, A. W. Alani and R. G. Alany, Drug Delivery, 2014, 21, 87-100.

54 J. Varshosaz, A. Pardakhty, V. I. Hajhashemi and A. R. Najafabadi, Drug Delivery, 2003, 10, 251-262.

55 M. Nasr, S. Mansour, N. D. Mortada and A. A. Elshamy, J. Microencapsulation, 2008, 25, 499-512.

56 E. Hood, M. Gonzalez, A. Plaas, J. Strom and M. VanAuker, Int. J. Pharm., 2007, 339, 222-230.

57 S. Zhang, H. Gao and G. Bao, ACS Nano, 2015, 9, 8655-8671. 58 L. Shang, K. Nienhaus and G. U. Nienhaus, J. Nanobiotechnol., 2014, 12, 5.

59 E. Frohlich, Int. J. Nanomed., 2012, 7, 5577-5591.

60 S. N. Manzoor Ahmad Gatoo, M. Y. Arfat, A. M. Dar, K. Qasim and S. Zubair, BioMed Res. Int., 2014, 1-8. 
61 M. S. Mohamed, S. Veeranarayanan, A. Baliyan, A. C. Poulose, Y. Nagaoka, H. Minegishi, S. Iwai, Y. Shimane, Y. Yoshida, T. Maekawa and D. S. Kumar, Macromol. Biosci., 2014, 14, 1696-1711.

62 Quality Analysis of traditional Chinese medicines, http:// qatcm.nricm.edu.tw/analysis.php?id $=2720$, accessed June 2018.

63 D. S. Shaker, M. A. Shaker and M. S. Hanafy, Int. J. Pharm., 2015, 493, 285-294.

64 S. Ullah, M. R. Shah, M. Shoaib, M. Imran, A. M. Elhissi, F. Ahmad, I. Ali and S. W. Shah, Drug Delivery, 2016, 23, 3480-3491.
65 S. Garcia-Salinas, E. Himawan, G. Mendoza, M. Arruebo and V. Sebastian, ACS Appl. Mater. Interfaces, 2018, 10, 1919719207.

66 J. M. Miller-Kleinhenz, E. N. Bozeman and L. Yang, Wiley Interdiscip. Rev.: Nanomed. Nanobiotechnol., 2015, 7, 797816.

67 J. Xue, R. Li, X. Zhao, C. Ma, X. Lv, L. Liu and P. Liu, Chem.Biol. Interact., 2018, 283, 59-74.

68 R. A. Naresh, N. Udupa and P. U. Devi, Indian J. Exp. Biol., 1996, 34, 764-772. 